\title{
Role of fibreoptic bronchoscopy in the diagnosis of mycobacterial diseases
}

\author{
A FUNAHASHI, GH LOHAUS, J POLITIS, LJ HRANICKA \\ From the Department of Medicine, Veterans Administration Medical Center, and the Medical College of \\ Wisconsin, Milwaukee, Wisconsin, USA
}

ABSTRACT From January 1976 to December 1981 mycobacteria were recovered for the first time from the respiratory tract of 179 patients. Twenty-three patients had undergone fibreoptic bronchoscopy during initial investigation after three or more expectorated sputum specimens were negative for acid-fast bacilli. Three of these patients had nodular lesions on the chest radiograph and the diagnosis of mycobacterial disease was made only after thoracotomy. In the remaining 20 patients bronchial brushings yielded a positive culture in 19 , while bronchial brushing was negative in one patient in whom culture of sputum before bronchoscopy had been positive. In eight of these 19 patients (group A) bronchial brushing was the only source that gave a positive result from culture, while in 11 patients (group B) both bronchial brushing and prebronchoscopy sputum yielded positive cultures. When these two groups were compared no difference was seen in their clinical presentation or radiographic findings but there was a notable difference in the quality of the prebronchoscopy sputum. Six of eight patients in group A had poor prebronchoscopy sputum, while 10 of 11 in group B had good prebronchoscopy sputum. It is concluded that, if a patient is unable to produce sputum or is able to produce only a poor specimen, fibreoptic bronchoscopy may be a useful means of obtaining additional material for culture.

The role of fibreoptic bronchoscopy in suspected mycobacterial disease has not been clearly defined. Recent investigations have shown that fibreoptic bronchoscopy may yield excellent material for mycobacterial culture. ${ }^{1-3}$ In these series, however, the sputum specimens obtained before the bronchoscopy also yielded positive cultures in a relatively large number of patients and this suggests the need for careful screening before fibreoptic bronchoscopy.

To evaluate the role of fibreoptic bronchoscopy further we reviewed our own experience during six years at the Wisconsin Veterans Administration Medical Center.

\section{Methods}

All records of acid-fast staining and culture for mycobacteria from January 1976 to December 1981

Address for reprint requests: Dr A Funahashi, Medical Service/ $111 \mathrm{E}$, Veterans Administration Medical Center, Wood (Milwaukee), Wisconsin 53193, USA.

Accepted 26 November 1982 in the tuberculosis laboratory were reviewed. We studied the clinical records and chest radiographs of patients who had undergone fibreoptic bronchoscopy and who had had one or more positive mycobacterial cultures from sputum or bronchoscopy specimens during the same period.

Sputum specimens submitted to the laboratory were routinely digested with $3 \%$ sodium hydroxyl for 20 minutes and then centrifuged at $3000 \mathrm{rpm}$. The sediments were stained by the aurominerhodamine fluorescence technique. An aliquot was inoculated into Lowenstein-Jensen media. Transbronchoscopic lung biopsy and surgical specimens were stained by the haematoxylin and eosin and Ziehl-Neelsen techniques. One or two biopsy specimens or a portion of the surgical specimens were cultured in the Lowenstein-Jensen media. The expectorated sputum was initially examined by an experienced technician, and the quality of the specimen was recorded. The specimen was recorded as "good" if it contained mucopurulent material of a brownish-yellow to greenish colour. The specimen was labelled "poor" if it contained primarily saliva.

Fibreoptic bronchoscopy was performed with the Olympus BF-B2 flexible bronchoscope under local 
anaesthesia. An oral endotracheal tube was used routinely, and the abnormal area on the chest radiograph was brushed with a standard Olympus brush under fluoroscopic guidance. The brush and bronchoscope were removed with the tip of the brush remaining outside the suction channel. After removal of the bronchoscope the brush was advanced into a container with $5 \mathrm{ml}$ of sterile saline and vigorously agitated to remove the material. Bronchial secretions were collected in a Luken's trap throughout the procedure. When an infectious process was the major consideration the specimen collected in the Luken's trap was not routinely sent for bacteriological or fungal studies. Bronchial secretions were used primarily for cytological examination.

Clinical records were examined for evidence of fever $\left(>37^{\circ} \mathrm{C}\right)$, increased cough or sputum production, weight loss $(>3 \mathrm{~kg})$, and skin tuberculin reactivity. The chest radiographs were examined and the location and extent of the lesion and the presence or absence of cavitation on the posteroanterior film were noted.

\section{Results}

During the period reviewed 179 patients had new positive cultures of mycobacterial species from material obtained from sputum, transtracheal aspirate, or bronchial brushings. Seventy-seven patients had cultures positive for Mycobacterium tuberculosis, 17 for $M$ kansasii, 84 for $M$ avium intracellulare, and one for $M$ fortuitum.

Forty-eight patients underwent fibreoptic bronchoscopy during the course of their illness (fig). Twenty-five patients had fibreoptic bronchoscopy for evaluation of haemoptysis or suspected coexisting neoplasm after expectorated sputum specimens had shown acid-fast bacilli on direct examination or the culture had yielded mycobacteria. Twenty-three patients underwent fibreoptic bronchoscopy as part of their initial investigation after three or more sputum smear specimens were negative for acid-fast bacilli. Three patients with negative cultures had nodular lesions visible on the chest radiograph and the diagnosis was made only after thoracotomy. Twenty of these 23 patients had positive cultures from either bronchial brushings or prebronchoscopy sputum and showed infiltrative lesions on the chest radiograph. To assess the diagnostic value of fibreoptic bronchoscopy, the positive yields of various specimens from these 20 patients were analysed. The results are summarised in table 1 . The culture of bronchial brushings was positive in 19 , while direct examination was positive in only one. In eight of the 20 patients bronchial brushings were the only source

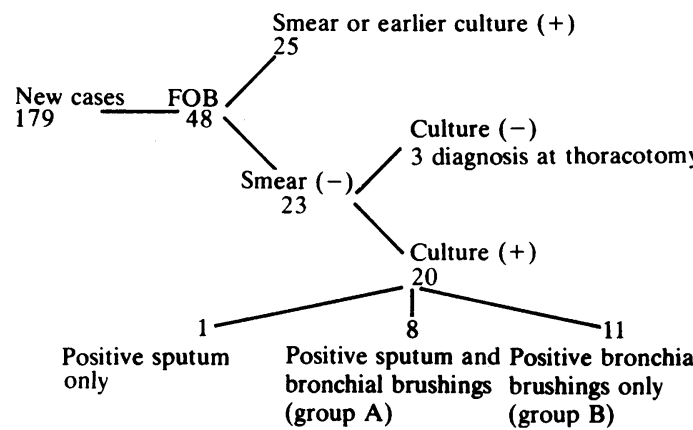

Results of mycobacterial study and fibreoptic bronchoscopy (FOB).

Table 1 Yield of evidence of mycobacterial infection from individual specimens

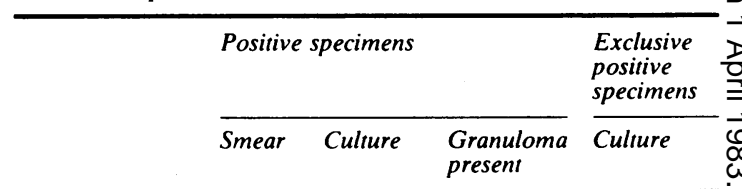

\begin{tabular}{|c|c|c|c|c|}
\hline $\begin{array}{l}\text { Bronchial brushing } \\
(n=20)\end{array}$ & 1 & 19 & & 8 \\
\hline $\begin{array}{l}\text { sputum } \\
(n=20)\end{array}$ & 0 & 12 & & 1 \\
\hline TBLB $(n=6)$ & 2 & $3^{*}$ & 4 & 0 \\
\hline
\end{tabular}

TBLB-transóronchoscopi: lung biopsy.

*Only five transbronchoscopic lung biopsy specimens were cultured.

of positive culture. Prebronchoscopy sputum specimens grew mycobacteria in 12 patients. Eleven of these 12 patients also had positive cultures from $\frac{0}{2}$ bronchial brushings, while in one patient prebron- $x$ choscopy sputum was the only source of a positive $\frac{5}{3}$ culture. Transbronchoscopic lung biopsy was performed in only six patients. Acid-fast bacilli were $O$ found in the biopsy material from two of these six patients, and cultures of tissue grew mycobacteria in $\mathrm{S}$ three cases. Granuloma was seen in four of the six patients. The 19 patients who had positive cultures from bronchial brushings were further divided into $N$ two groups. Group A consisted of eight patients in whom bronchial brushings were the only source of positive culture, and group B consisted of $11 \mathrm{\omega}$ patients in whom both bronchial brushings and? prebronchoscopy sputum were culture positive Table 2 shows the type of organism cultured in both groups. When clinical characteristics were com-pared, there was no apparent difference in the presence of symptoms or the prevalence of a reaction to $\frac{\mathrm{O}}{\overrightarrow{\mathrm{D}}}$ tuberculin skin tests (table 3 ). In both groups the? lesions seen on the chest radiograph were generally⿺ unilateral and limited to the upper lobe, except in two patients who had a miliary pattern. When a cav 
Table 2 Species of mycobacteria cultured

\begin{tabular}{|c|c|c|c|c|}
\hline Group* & $\begin{array}{l}M \\
\text { tuberculosis }\end{array}$ & $M$ kansasii & $\begin{array}{l}M \text { avium } \\
\text { intracellulare }\end{array}$ & Total \\
\hline $\begin{array}{l}\text { A } \\
\text { B }\end{array}$ & $\begin{array}{l}4 \\
7\end{array}$ & $\begin{array}{l}2 \\
0\end{array}$ & $\begin{array}{l}2 \\
4\end{array}$ & $\begin{array}{r}8 \\
11\end{array}$ \\
\hline
\end{tabular}

Group A: Prebronchoscopy sputum subsequently negative on culture, bronchial brushings positive; group B: prebronchoscopy sputum and bronchial brushings both positive on culture.

Table 3 Comparison of clinical characteristics in 19 patients with positive cultures of mycobacteria from bronchial brushings

\begin{tabular}{lll}
\hline & $\begin{array}{l}\text { Group } A^{*} \\
(n=8)\end{array}$ & $\begin{array}{l}\text { Group } B^{*} \\
(n=11)\end{array}$ \\
\hline $\begin{array}{l}\text { Symptoms present } \\
\text { Tuberculin positive }\end{array}$ & 4 & 5 \\
$\begin{array}{l}\text { Radiographic evidence } \\
\text { of cavity }\end{array}$ & 6 & 6 \\
$\begin{array}{l}\text { Sputum of good quality } \\
\text { Sput }\end{array}$ & 4 & 6 \\
\hline
\end{tabular}

${ }^{*}$ Groups defined in footnote to table 2.

ity was present, it was smaller than $1.5 \mathrm{~cm}$ in diameter in all but one. There was no difference between groups in terms of the extent of radiographic abnormality or the presence or absence of a cavity. Only two of eight patients in group A had sputum specimens considered to be of good quality, while 10 of the 11 patients in group $B$ had good sputum specimens.

\section{Discussion}

In 1979 Danek and Bower ${ }^{1}$ reported their experience with fibreoptic bronchoscopy in active pulmonary tuberculosis. They obtained positive cultures from material obtained by fibreoptic bronchoscopy in 39 of 41 patients. In the present series 19 of 20 patients with an infiltrative process evident on the chest radiograph had a positive culture from bronchial brushings. Fibreoptic bronchoscopy therefore provides excellent material for culture when there is active mycobacterial disease. Furthermore, in eight $(40 \%)$ of our patients and $19(46 \%)$ in the series of Danek and Bower the positive culture of mycobacteria was obtained only from the specimens obtained by fibreoptic bronchoscopy. The diagnosis of mycobacterial disease would probably not have been made had fibreoptic bronchoscopy not been performed. Two of our patients in group A had positive cultures for $M$ avium intracellulare only from bronchial brushings. They probably represent saprophytic colonisation rather than active disease. Four patients in group B had multiple positive sputum cultures in addition to positive bronchial brushings and were thought to have active $M$ avium intracellulare disease. ${ }^{4}$
The low yield of positive results from examination of smears from bronchial brushings was not unexpected. We had only one positive smear from 19 patients who subsequently had positive cultures from bronchial brushings. Brushing alone therefore is of limited value. Recently Wallace $e t a l^{2}$ emphasised the value of an immediate diagnosis by fibreoptic bronchoscopy and transbronchial lung biopsy. They showed acid-fast bacilli or caseating granulomas in 11 of 23 patients. Although we have only six patients who had transbronchial lung biopsy with fibreoptic bronchoscopy, four of these six patients showed granuloma in transbronchial lung biopsy specimens. Fibreoptic bronchoscopy with transbronchial lung biopsy may be quite useful when the diagnosis is urgently needed.

In less acute cases the indication for fibreoptic bronchoscopy is less clear. A relatively large number of patients in our series, as well as in previously reported studies, yielded positive culture results from sputum specimens obtained before fibreoptic bronchoscopy. ${ }^{1-3}$ This suggests that selection of patients for fibreoptic bronchoscopy is desirable. Comparison of the group of patients with a positive culture only from bronchial brushings (group A) and the group of patients with positive cultures from both prebronchoscopy sputa and bronchial brushings (group B) showed no clear-cut differences in their clincial and radiographic features. This is probably because the disease process was limited in most of our patients. Most patients in group A, however, had sputum specimens of very poor quality, while most patients in group B had sputum specimens of good quality. It appears that fibreoptic bronchoscopy is indicated in patients who are unable to produce sputum or produce very poor samples. In patients who are able to produce mucopurulent sputum its place is questionable, since in most of the patients eventually confirmed as suffering from mycobacterial disease the subsequent culture is likely to yield mycobacteria.

In conclusion, firstly, bronchial brushing performed via the fibreoptic bronchoscope can provide excellent material for mycobacterial culture. Secondly, fibreoptic bronchoscopy combined with transbronchial lung biopsy may provide early diagnosis of mycobacterial disease when establishment of the diagnosis is urgent. Finally, fibreoptic bronchoscopy is useful in patients who are unable to produce sputum or produce very poor specimens for culture, but may be deferred in patients producing mucopurulent sputum.

The authors wish to thank Mr Joseph Diageneault, tuberculosis laboratory technologist, for his invaluable assistance. 


\section{References}

' Danek SJ, Bower JS. Diagnosis of pulmonary tuberculosis by flexible fiberoptic bronchoscopy. Am Rev Respir Dis 1979;119:677-9.

${ }^{2}$ Wallace JM, Deutsch AL, Harrell JH, Moser KM. Bronchoscopy and transbronchial biopsy in evaluation of

\section{Notices of books received}

A Colour Atlas of Chest Trauma and Associated Injuries, vol 1. Augustin Besson and Frederic Saegesser. (Pp 384; £48.) Wolfe Medical Publications Limited, 3 Conway Street, London.

This is the first volume of a two-volume work intended to provide a comprehensive guide to the nature and management of all types of chest injury. When the second volume is available it is likely to become the most important work on the subject that is currently available. Although described as an atlas the book gives a very detailed and complete account of the management of all types of chest injury and is superbly illustrated with diagrams, photographs, radiographs, and colour illustrations of pathological injuries and their management. The work is based largely on clinical experience at the Centre Hospitalier Universitaire Vaudois, Lausanne, Switzerland. Volume 2 patients with suspected active tuberculosis. Am J Med 1981;70:1189-94.

${ }^{3}$ Jett JR, Cortese DA, Dines DE. The value of bronchoscopy in the diagnosis of mycobacterial disease. Chest 1981;80:575-8.

4 Yammamoto M, Ogura Y, Sudo K, et al. Diagnostic criteria for disease caused by atypical mycobacteria. Am Rev Respir Dis 1967;96:773-8.

is intended to cover trauma of the trachea, oesophagus, $\stackrel{\sim}{\sigma}$ diaphragm, heart, and great vessels. The work should be regarded as a standard reference source for all those con- $\triangle$ cerned with the handling of major injuries.

Cardiac Bioprostheses: Proceedings of the Second International Symposium. Lawrence $\mathbf{H}$ Cohn and Vincenzo $\overrightarrow{0}$ Gallucci. (Pp 591; \$70.) Yorke Medical Books, 875 Third Avenue, New York City. 1982.

This book is a record of the proceedings of the second international symposium on cardiac bioprostheses held in Rome in May 1982. Papers submitted for the meeting were

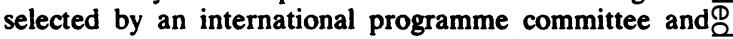
those which were presented have been edited for publication in this work. The subjects covered include clinical analysis, haemodynamics, thromboembolism, paediatric valve replacement, experimental pathology, explant pathology, bioprosthetic bioengineering, and long-term follow-up. 